\title{
Avaliação de parsers na detecção de relações essenciais do modelo Universal Dependencies para o português
}

\author{
Luana Balador Belisário, Thiago Alexandre Salgueiro Pardo \\ Núcleo Interinstitucional de Linguística Computacional (NILC) \\ Instituto de Ciências Matemáticas e de Computação, Universidade de São Paulo \\ São Carlos, Brasil
}

\begin{abstract}
Resumo. Este artigo descreve o estudo do desempenho de dois parsers conhecidos para o português com base nas diretrizes do modelo internacional "Universal Dependencies". Visando mapear o estado da arte na área, os parsers foram avaliados com relação à detecção de algumas relações essenciais do modelo que indicam os argumentos principais dos verbos. Mostramos que o parser UDPipe se destaca entre os parsers avaliados, mas que ainda há muito a avançar na área.
\end{abstract}

\section{Introdução}

O modelo Universal Dependencies ${ }^{1}$ (UD) (Nivre, 2015; Nivre et al., 2020) é uma proposta internacional para anotação "universal" morfossintática e sintática (incluindo características morfológicas, classes gramaticais e dependências sintáticas) de sentenças em diferentes idiomas. A iniciativa já conta com mais de 300 contribuidores, produzindo quase 200 treebanks anotados com as diretivas definidas do modelo para mais de 100 idiomas. A Figura 1 ilustra a anotação de uma sentença em português (reproduzida de Rademaker et al., 2017, p. 200). Pode-se ver a sentença original com suas palavras conectadas por relações de dependência sintática acima, assim como os lemas e as etiquetas morfossintáticas abaixo.

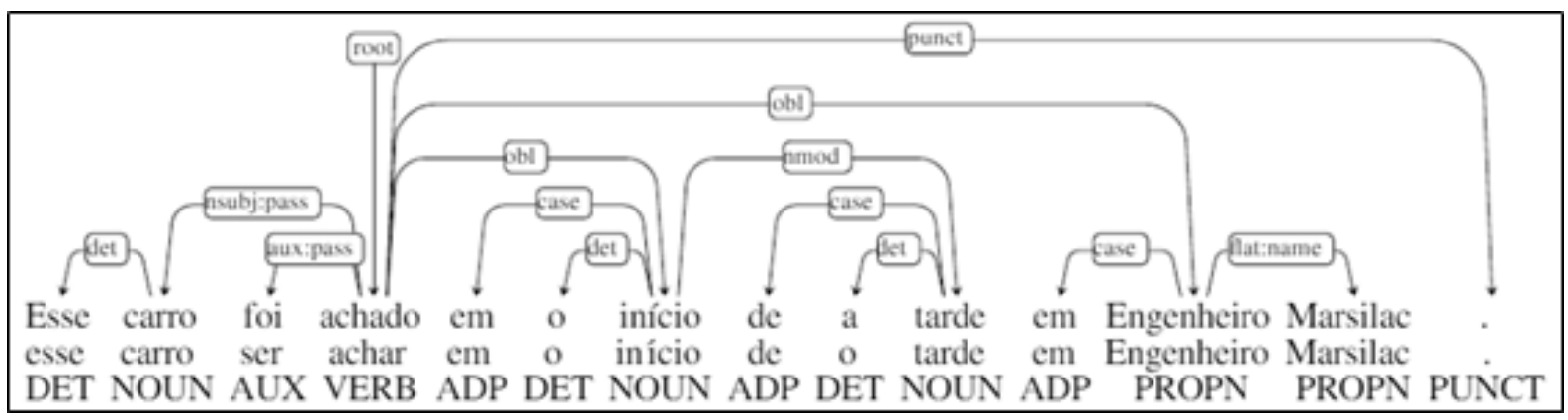

Figura 1. Exemplo de sentença em português anotada segundo o modelo UD.

Em função da grande adesão ao modelo UD e sua utilidade para o desenvolvimento de aplicações de Processamento de Linguagem Natural (PLN), taggers e parsers com base em UD têm sido criados para diversas línguas. Para o português, há alguns parsers que se destacam pelo uso na comunidade de pesquisa e que são objeto de análise neste trabalho, em

\footnotetext{
${ }^{1}$ https://universaldependencies.org/
} 
especial, o UDPipe (Straka, 2018) e o PassPort (Zilio et al., 2018). Em seus trabalhos originais, os autores relatam valores de desempenho geral na ordem de 85 a $87 \%$ para anotação de relações sintáticas.

O objetivo deste trabalho é verificar o desempenho dos dois parsers citados de forma mais pontual, calculando precisão, cobertura e medida-f para algumas relações ditas essenciais (core) que denotam os argumentos centrais dos verbos, a saber: 'nsubj' (nominal subject), 'obj' (object) e 'iobj' (indirect object). Inspirada por outras iniciativas (por exemplo, Collovini et al., 2018, e Gonçalves et al., 2020), essa proposta faz parte de um esforço de mensurar de forma mais concreta os pontos fortes e fracos dos sistemas, visando fornecer subsídios para futuras pesquisas na área.

A seguir, descrevemos brevemente a metodologia adotada, sendo que os resultados obtidos são sintetizados na Seção 3. A Seção 4 apresenta algumas considerações finais.

\section{Metodologia}

\subsection{O córpus de teste}

O córpus Bosque foi anotado sintaticamente segundo as diretrizes da UD (como relatam Rademaker et al., 2017) por um grupo de pesquisadores da área e é utilizado nesse artigo como córpus de referência (gold standard), para avaliar a acurácia dos parsers testados. $\mathrm{O}$ córpus é composto por 9.364 sentenças e 210.957 tokens.

\subsection{Um novo tokenizador}

Além da versão padrão de tokenização disponibilizada com cada parser, também se utilizou um novo tokenizador desenvolvido no âmbito deste trabalho, mais alinhado com as diretrizes da UD, visando-se avaliar seu impacto na tarefa. O novo tokenizador, chamado LBTokenizer ${ }^{2}$, pode ser utilizado sozinho ou integrado ao UDPipe.

\subsection{A ferramenta de avaliação}

O Conllu-File-Comparator ${ }^{3}$ é um software desenvolvido na linguagem Python para essa pesquisa que compara as ocorrências das relações 'nsubj', 'obj' e 'iobj' de um arquivo com sentenças anotadas automaticamente pelos parsers com suas versões de referência, sendo que as anotações devem estar no formato CoNLL-U, amplamente adotado na área. Esse formato consiste em um conjunto de informações tabeladas, em que as palavras de uma sentença estão nas linhas e cada coluna armazena um tipo diferente de informação sobre as palavras.

Para cada sentença do arquivo de referência, o software contabiliza o número de cada relação essencial presente na sentença e calcula as medidas de precisão, cobertura e medida-f (assim como seus desvios padrões) para cada uma delas. Por exemplo, vamos calcular a precisão, cobertura e medida-f para uma sentença de teste com cinco tokens cujas relações essenciais estão representadas na Figura 1 e a sentença de referência com sua estrutura de relações representada na Figura 2 (note que, para simplificar a demonstração, omitimos as relações não essenciais e trocamos os tokens por números).

\footnotetext{
${ }^{2}$ Disponível em https://github.com/Lubelisa/LBTokenizer-UDPipe

${ }^{3}$ Disponível em https://github.com/Lubelisa/Conllu-File-Comparator
} 


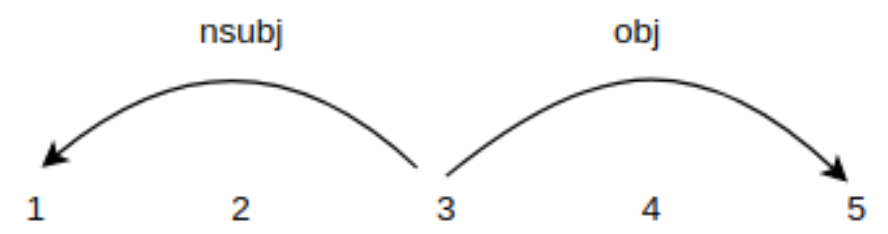

Figura 1. Sentença de teste, podendo ser anotada pelo UDPipe ou pelo PassPort

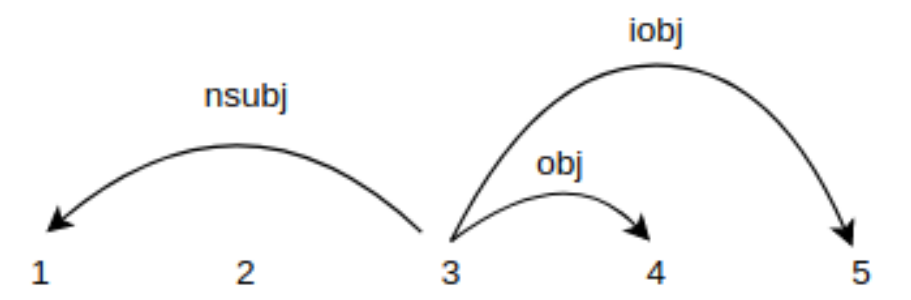

Figura 2. Sentença de referência, com anotação feita/revisada por um especialista

$\mathrm{Na}$ sentença de referência, há um caso de cada relação essencial e, na sentença de teste, um caso de relação 'nsubj' e um caso de 'obj'. Para calcular a precisão, precisa-se contabilizar a porcentagem de relações da sentença de teste que estão de acordo com o previsto na sentença de referência. Por exemplo, para calcular a precisão da relação 'nsubj' na sentença de teste, é necessário utilizar a fórmula a seguir.

Precisão ${ }_{n s u b j}=\frac{\text { número de relações 'nsubj' na sentença de teste que estão de acordo com a referência }}{\text { número de relações 'nsubj' na sentença de teste }}$

Assim, para a sentença de teste da Figura 1, o valor da precisão para a relação 'nsubj' é dada por Precisão ${ }_{n s u b j}=\frac{1}{1}=1$ ou 100\%. A precisão para a relação 'obj' é zero, pois, apesar de haver na sentença de teste um caso de 'obj', esse caso não é entre os mesmos tokens da sentença de referência e na mesma ordem (do token 3 para o 5).

No cálculo da cobertura, o denominador da fórmula verifica o número de relações da sentença de referência, ou seja, divide-se o número de cada relação essencial da sentença de teste que esteja de acordo com a sentença de referência pelo número de relações essenciais na sentença de referência. Logo, para a relação 'nsubj', a fórmula é:

Cobertura $_{n s u b j}=\frac{\text { número de relações 'nsubj' na sentença de teste que estão de acordo com a referência }}{\text { número de relações 'nsubj' na sentença de referência }}$

Para a sentença de teste da Figura 1, o valor da cobertura para a relação 'nsubj' se dá por Cobertura $_{n s u b j}=\frac{1}{1}=1$ ou $100 \%$. Caso houvesse dois casos de relação 'nsubj' na sentença de referência, o valor da cobertura para a relação seria de 0,5 ou $50 \%$, portanto. 
Por fim, a medida-f é uma combinação da precisão e da cobertura de cada relação, com o objetivo de se ter uma métrica única de avaliação. Foi utilizada a fórmula a seguir para calcular a medida-f de cada relação essencial:

$$
\text { Medida }-f_{\text {relação }}=\frac{2 * \text { Precisão } o_{\text {relacão }}{ }^{*} \text { Cobertura } a_{\text {relação }}}{\text { Precisão }{ }_{\text {relação }}+\text { Cobertura } a_{\text {relaçao }}}
$$

A seguir, relatamos os resultados obtidos para os dois parsers avaliados.

\section{Resultados}

O procedimento para a realização dos testes com os parsers foi passar por eles as sentenças de teste do córpus Bosque e comparar o arquivo de saída - que contém as sentenças anotadas automaticamente - com o arquivo de referência do Bosque que foi revisado por especialistas. Essa comparação e o cálculo dos resultados foi feito pelo software Conllu-File-Comparator e foram realizados três testes com os parsers: (1) utilizando o UDPipe para tokenizar e anotar as sentenças, (2) utilizando o LBTokenizer para tokenizar e o UDPipe apenas para anotar e (3) utilizando o PassPort para tokenizar e anotar as sentenças (pois a ferramenta foi desenvolvida para realizar as etapas em conjunto e não foi possível isolá-las). Os resultados médios dos testes (medidas e desvios padrões - DP) são mostrados na Tabela 1.

Tabela 1. Resultados dos parsers

\begin{tabular}{|c|c|c|c|c|c|c|c|c|c|}
\hline & \multicolumn{3}{|c|}{ UDPipe } & \multicolumn{3}{c|}{ LBTokenizer+UDPipe } & \multicolumn{4}{c|}{ PassPort } \\
& nsubj & obj & iobj & \multicolumn{1}{|c|}{ nsubj } & obj & iobj & nsubj & obj & iobj \\
\hline Precisão & 0,82 & 0,85 & 0,73 & 0,48 & 0,54 & 0,33 & 0,35 & 0,41 & 0,37 \\
\hline Cobertura & 0,80 & 0,76 & 0,29 & 0,46 & 0,48 & 0,10 & 0,66 & 0,47 & 0,33 \\
\hline Medida-f & 0,81 & 0,80 & 0,41 & 0,47 & 0,50 & 0,15 & 0,46 & 0,44 & 0,35 \\
\hline DP Precisão & 0,33 & 0,30 & 0,42 & 0,46 & 0,45 & 0,47 & 0,13 & 0,37 & 0,17 \\
\hline DP Cobertura & 0,36 & 0,37 & 0,44 & 0,46 & 0,45 & 0,28 & 0,21 & 0,41 & 0,35 \\
\hline
\end{tabular}

Analisando-se as tabelas, conclui-se que o UDPipe sozinho obteve o melhor resultado. Para as relações 'nsubj' e 'obj', o UDPipe atingiu $80 \%$ de medida-f, mas ainda abaixo dos $87 \%$ relatados no artigo original (que engloba a avaliação de todas as relações). Há, portanto, uma grande margem para melhoria do sistema, o que se torna muito importante quando se considera que a análise produzida pelo parser é a entrada para outros processos em aplicações de PLN. Um erro nessa etapa pode ser propagado em outras, podendo impactar significativamente os resultados almejados. Também chama a atenção o baixo desempenho do PassPort para as três relações essenciais, apesar de, na maioria dos casos, esse sistema apresentar os menores desvios padrões para as medidas. É interessante notar que todos os sistemas avaliados têm desempenho menor para a relação 'iobj', que é sabidamente um dos casos mais desafiadores na anotação linguística com o modelo UD.

Nota-se que o uso do LBTokenizer degradou os resultados do UDPipe. Isso ocorreu porque o Bosque não segue algumas diretrizes mais atuais da UD, e usar o LBTokenizer gerou sequências de palavras diferentes das utilizadas para o treinamento do UDPipe para o português. 


\section{Considerações finais}

Esse artigo apresenta um esforço em detalhar o desempenho de alguns parsers baseados no modelo UD para o português. Os resultados mostram que ainda é necessário avançar nessa frente. Trabalhos futuros incluem a avaliação de outras relações de dependência sintática e também o teste de outros sistemas, como o UDify (Kondratyuk and Straka, 2019).

Esse trabalho faz parte do projeto maior POeTiSA (POrtuguese processing - Towards Syntactic Analysis and parsing). Mais detalhes podem ser encontrados no portal web do projeto, em https://sites.google.com/icmc.usp.br/poetisa.

\section{Agradecimentos}

Os autores agradecem ao Centro de Inteligência Artificial da USP (C4AI https://c4ai.inova.usp.br/), que conta com o apoio da IBM e da FAPESP (\#2019/07665-4), e à Universidade de São Paulo pelo suporte financeiro.

\section{Referências}

Collovini, S.; Santos, H.D.P.; Lima, T.; Fonseca, E.; Pereira, B.; Souza, M.; Moraes, S.; Vieira, R. (2018). Cross-Framework Evaluation for Portuguese POS Taggers and Parsers. 19th International Conference on Computational Linguistics and Intelligent Text Processing (CICLing).

Gonçalves, M.; Coheur, L.; Baptista, J.; Mineiro, A. (2020). Avaliação de recursos computacionais para o português. LinguaMÁTICA, Vol. 12, N. 2, pp. 51-68.

Kondratyuk, D. and Straka, M. (2019). 75 Languages, 1 Model: Parsing Universal Dependencies Universally. In the Proceedings of the Conference on Empirical Methods in Natural Language Processing and the International Joint Conference on Natural Language Processing, pp. 2779-2795.

Nivre, J. (2015). Towards a Universal Grammar for Natural Language Processing. In the Proceedings of the 16th International Conference on Intelligent Text Processing and Computational Linguistics (CICLing), pp. 3-16.

Nivre, J.; Marneffe, M-C.; Ginter, F.; Hajič, J.; Manning, C.D.; Pyysalo, S.; Schuster, S.; Tyers, F.; Zeman, D. (2020). Universal Dependencies v2: An Evergrowing Multilingual Treebank Collection. In the Proceedings of the 12nd International Conference on Language Resources and Evaluation (LREC), pp. 4034-4043.

Rademaker, A.; Chalub, F.; Real, L.; Freitas, C.; Bick, E.; Paiva, V. (2017). Universal Dependencies for Portuguese. In the Proceedings of the 4th International Conference on Dependency Linguistics (Depling), pp. 197-206.

Straka, M. (2018). UDPipe 2.0 prototype at CoNLL 2018 UD shared task. In the Proceedings of the CoNLL Shared Task: Multilingual Parsing from Raw Text to Universal Dependencies, pp. 197-207.

Zilio, L.; Wilkens, R.; Fairon, C. (2018). PassPort: A Dependency Parsing Model for Portuguese. In the Proceedings of the 13rd International Conference on the Computational Processing of Portuguese (PROPOR), pp. 479-489. 